\title{
Using protein microarray reveals clinical correlation between self-perception of patient and the apoptosis-related proteins in rheumatoid arthritis
}

Jian-ting Wen

Anhui University of Traditional Chinese Medicine

Jian Liu (D liujianahzy@126.com )

Anhui University of Traditional Chinese Medicine

Hui Jiang

Anhui University of Traditional Chinese Medicine

Lei Wan

Anhui University of Traditional Chinese Medicine

Ling Xin

Anhui University of Traditional Chinese Medicine

Yue Sun

Anhui University of Traditional Chinese Medicine

Yan-qiu Sun

Anhui University of Traditional Chinese Medicine

Ying Zhang

Anhui University of Traditional Chinese Medicine

Xin-lei Du

Anhui University of Traditional Chinese Medicine

Xin Wang

Anhui University of Traditional Chinese Medicine

Jie Wang

Anhui University of Traditional Chinese Medicine

Research

Keywords: rheumatoid arthritis, proteins, apoptosis, SPP

Posted Date: July 2nd, 2020

DOI: https://doi.org/10.21203/rs.3.rs-39433/v1 
License: (c) (i) This work is licensed under a Creative Commons Attribution 4.0 International License. Read Full License 


\section{Abstract}

Background: The most severe effects of rheumatoid arthritis (RA) are loss of physical function, which may have a significant impact on self-perception of patient (SPP). However, the inherent relationship between SPP and the key proteins is not clear. The aim of this study was to get an insight into SPP of RA in connection with the the apoptosis-related proteins.

Methods: We set out to investigate changes of the apoptosis-related proteins expression in the peripheral blood mononuclear cells (PBMCs) of RA. Additionally, we aimed to correlate the apoptosis-related proteins expression profiles with SPP and clinical indexes. To this end, we employed antibody microarrays of the the apoptosis-related proteins in PBMCs from four RA patients and seven healthy controls. We used bioinformatics to screen several the apoptosis-related proteins. To validate key protein candidates, we performed Enzyme linked immunosorbent assay (ELISA) on 30 RA patients and 30 healthy controls.

Results: We found the expression of ten the apoptosis-related proteins (caspase3, CD40, SMAC, HSP27, HTRA, IGFBP-1, IGFBP-6, sTNF-R1, sTNF-R2, TRAILR-3) were significantly altered in PBMCs of RA patients. Receiver operating characteristic (ROC) curve analysis suggested that these ten the apoptosisrelated proteins are potential biomarkers of RA. Spearman Correlation analysis and Logistic-regression analysis revealed that the 10 selected the apoptosis-related proteins correlated with SPP and clinical indexes.

Conclusion: Therefore, we highlight some the apoptosis-related proteins may serve as potential biomarkers in prediction of SPP for RA patients, although the underlying mechanisms need to be further explored.

\section{Background}

RA is an autoimmune disease and the most common type of inflammatory arthritis that is a major cause of disability ${ }^{12}$. The overall world prevalence of rheumatoid arthritis (RA) ranges from $0.5-1.0 \%$, women are 2-3 times more likely to experience major depression than $\mathrm{men}^{3}{ }^{4}$. It was eventually lead to joints deformity and loss of function if not adequately treated ${ }^{5}$. RA patients not only suffer from physical pain, but also bear great psychological pressure ${ }^{67}$. A decrease in functionality is accompanied by deteriorating social and emotional well-being ${ }^{8}$. There, persistent psychological stress often leads to anxiety disorders, depression, and quality of life (QOL) decreased ${ }^{9}{ }^{10}$. In particular, SPP has attracted more and more attention in the clinical which closely related to the therapeutic effect. Currently, the internationally recommended that SPP of RA is evaluated using DAS28, VAS, SAS, SDS, SF-36 and there has become the most accepted evaluation method by the majority of clinicians ${ }^{11}$. A previous study performed a crosssectional study with RA patients in Ecuador, they found that the QOL of RA patients is severely affected, depression, fatigue, morning stiffness, pain, high disease activity, and disability have a negative effect on 
QOL in RA, likewise, patients with more comorbidities and extraarticular manifestations show worse $\mathrm{QOL}^{12}$.

The exact causes of in RA is unknown. Recently, the number of studies revealing the important role of epigenetics in the pathogenesis of RA has increased ${ }^{13} 14$. However, we do not know whether it is related to decreased SPP in RA. These days, although there are advances in the treatment of RA, we still need to discovery the pathogenesis of RA. The RA clinical diagnosis is mainly based on causes of RA, clinical symptoms, signs, laboratory tests, and clinical imaging ${ }^{15}$. Until now there is no gold standard for early diagnostic approach for RA, and patients are usually comprehensively evaluated by serological tests for rheumatoid factor (RF) and anti-citrullinated protein antibodies (ACPA) and for inflammatory markers such as hypersensitive C-reactive protein (CRP), an erythrocyte sedimentation rate (ESR) test, and an imaging test if symptoms appear ${ }^{16} 17$. However, RF-positive rate and ACPA-specificity can be detected in approximately $50-80 \%$ of RA patients, which suggesting that the overall detection efficiency is lower ${ }^{18}$. Apoptosis plays a key role in RA, and the inhibition of apoptosis may provide a novel treatment method for RA diseases ${ }^{19} 20$. Therefore, there is an increasingly growing interest in identifying biomarkers for diseases, in which apoptosis is involved. Studies toward novel diagnostic biomarker discovery, using protein microarray assay, have been increasing ${ }^{21} 22$. A proteomics approach using protein microarray assays can be used to determine the pattern of proteins and compare their absolute levels between diseased and healthy controls ${ }^{23}$.

In present study, we obtained peripheral blood mononuclear cells (PBCMs) from four RA patients and tried to discover diagnostic biomarkers in informative blood samples using protein microarray assays. In the discovery and validation set, candidate biomarkers were selected and verified. Additionally, the relationship between these candidate biomarkers with SPP and clinical indexes of RA patients was also analyzed.

\section{Materials And Methods}

\section{RA patients and healthy controls}

Four patients (three females and one male, 45-66 years of age) with RA were recruited from the Department of Rheumatology and Immunology of First Affiliated hospital of Anhui University of Traditional Chinese Medicine from June 2019 to December 2019. Seven healthy control (five females and two males, 45-66 years of age) without previous history selected from the Physical Examination Center in First Affiliated hospital of Anhui University of Traditional Chinese Medicine. These RA patients and healthy controls samples (frst cohort) were used for the protein microarray and the associated ELISA verifcation.

We further recruited more RA patients and healthy controls samples per each group (second cohort) for the independent ELISA verifcation experiments (30 RA patients and 30 healthy controls). All RA patients fulfilled the 2010 ACR/EULAR (American College of Rheumatology/European League Against 
Rheumatism) criteria for the classification of $\mathrm{RA}^{24}$. In addition, 30 age- and sex-matched healthy controls with no clinical history of tumors, trauma, infectious diseases or autoimmune diseases, who underwent routine Physical Examination Center in the same hospital during the same period. All samples from RA patients and healthy controls were obtained with written informed consent. This study was approved by the ethics committee of the First Affiliated hospital of Anhui University of Traditional Chinese Medicine.

\section{Measurements}

We collected demographic and clinical data from the subjects, including age, gender, disease duration, blood chemistry. All clinical measurements were performed by the clinical laboratory staff of our hospital. The clinical laboratory data such as erythrocyte sedimentation rate (ESR), high-sensitivity C-reactive protein (CRP), rheumatoid factor (RF), anti-cyclic citrullinated peptide antibody (CCP) and clinical characteristics are determined.

All of the participants enrolled were asked to fill in the DAS28, VAS, SDS, SAS, SF-36 under the guidance of clinical doctors, SF-36 consists of 8 dimensions, namely physical functioning (PF), role-physical (RP), body pain (BP), general health (GH), vitality (VT), social functioning (SF), role-remotional (RE), mental health $(\mathrm{MH})$.

\section{PBMCs preparation and total RNA extraction}

An amount of $5 \mathrm{~mL}$ of whole blood was obtained from RA patients and healthy controls and PBMCs were isolated through Ficoll-Paque density gradient centrifugation (GE Healthcare, Uppsala, Sweden). The concentration of cells was adjusted to $5-7 \times 10^{6}$ cells per $\mathrm{ml}$ and reserved at $-80^{\circ} \mathrm{C}$ until use.

Total RNA was extracted from PBMCs of all samples using Trizol reagent (Invitrogen Life Technologies, Carlsbad, CA, USA) according to the manufacturers instructions. The RNA was then reverse transcribed to single stranded CDNA, which was used as a template to synthesize the second CDNA strand. Aliquots of total RNA samples were used to determine the RNA concentration and purity using the Nano Drop ND1000 spectral photometer (peqlab). The RNAs were selected on the basis of a combination of p-value, fold change, raw intensity and type. In addition, RNAs with miRNA response elements (MREs) related to RA reported in the literatures were selected preferentially. All qPCR assays were performed on the Viia7 Real-Time PCR System, each sample was replicated three times. Library quality was assessed on the Agilent Bioanalyzer 2100 system. The relative expression levels of RNAs were calculated using the $2-\Delta \Delta \mathrm{Ct}$ method.

\section{The apoptosis-related protein microarray analysis}

Capture antibodies were spotted onto a glass slide at a volume of $350 \mathrm{pL}$ per spot at a pitch of $500 \mathrm{~mm}$ using a microarray printer ${ }^{+}$. Biotinylated goat anti-bovine lgG antibody was also spotted as a detection control. Each capture antibody was printed in quadruplicate spots within a subarray. Each glass slide contained 16 identical subarrays separated by a 16-well gasketed hybridization chamber to prevent 
sample cross-contamination. Antibody arrays were stored at $-20^{\circ} \mathrm{C}$ until use. The raw signal data was extracted using MapPix 6.0 software and quantitative data were extracted and analyzed with software for the multiplexed antibody array.

\section{Bioinformatics Analysis}

The raw data obtained by the chip scan was subjected to chip background removal and inter-chip normalization processing by Raybiotech software.2.3.2. After the raw data were normalized by the software, the resulting data were selected for analysis. DEPs with $\mathrm{P}<0.05$ were first retained and then further screened by Foldchange (expression difference multiple). The selection conditions were as follows: $\otimes$ Foldchange $\leq 0.83$ or Foldchange $\geq 1.2$; and $\otimes$ Fluorescent signal $>150$. For cluster analysis, the heatmap. 2 function and gplots package from R/bioconductor were used. The distance between two samples was calculated as the Euclidean distance; the distance between the two clusters was calculated with the furthest neighbor method (complete), and the distance between classes was defined as the maximum distance. Fisher's exact test and the clusterProfiler package from R/bioconductor were used. For selection, the number of genes that differed on a certain GO term and KEGG pathway were $\geq 2$, and $P$ $<0.05$. The normalized data were exported to SIMCA-p 11.5 for principal component analysis (PCA), partial least-squares discriminant analysis (PLSDA), and orthogonal partial least-squares discriminant analysis (OPLSDA).

\section{Validation with ELISA}

According to the manufacturers'instructions, the differentially expressed proteins levels in serum were quantified using ELISA kit (eBioscience, Inc., San Diego, CA, USA). Briefly, whole-blood samples were centrifuged at $4,000 \mathrm{~g}$ for $10 \mathrm{~min}$ to collect serum. After that, conditioned media or supernatants of lysed samples were collected and added to 96-well plates pre-coated with appropriate capture antibody, followed by incubation with the appropriate biotinylated detection antibody. Lastly, Streptavidin horseradish-peroxidase (HRP) was added to each well and incubated for $1 \mathrm{~h}$ at room temperature. The results were detected using an ELISA microplate reader (BioTek) at $450 \mathrm{~nm}$.

\section{Statistical Analysis}

SPSS 23.0 software for Windows was used for statistical analysis. Continuous variables are expressed as means \pm standard deviation and categorical variables are expressed as numerals. When normally distributed, independent samples will be compared for numerical variables between the two groups using Student's t-test. Mann-Whitney U-test was used for the data not conforming to a normal distribution. Categorical data were compared between groups using the Chi-square test. Correlations between the variables were performed using Spearman Correlation Analysis and Logistic-regression. A p value $<0.05$ was statistically significant $\left({ }^{\star} p<0.05,{ }^{* \star} p<0.01,{ }^{\star \star \star} p<0.001\right)$.

\section{Results}


Basic characteristics of RA patients and healthy controls

Thirty RA patients ( 2 males, 28 females, mean age: $55.63 \pm 11.41$ years, course of disease: $15.67 \pm$ 8.53 years) and thirty healthy controls ( 2 males, 28 females, mean age: $54.33 \pm 12.59$ years) were used as the study group. No significant differences in age or gender were identified between the two groups ( $p>$ 0.05).

\section{Comparisons of SPP and clincial indexes between RA patients and healthy controls}

As showed in Table 1, compared with healthy control, PF, RP, GH, MH of RA were significantly lower, while DAS28, VAS, SAS, SDS, BP were significantly higher than healthy control $(P<0.05)$. Meanwhile, ESR, CRP, $R F, C C P$ of RA were significantly higher than $H C(P<0.05)$.

Table 1

Comparison of SPP and clincial data between the two groups

\begin{tabular}{|llll|}
\hline Index & RA $(\mathbf{n}=30)$ & Control $(\mathbf{n}=30)$ & $\mathbf{P}$ \\
\hline DAS28 score & $6.54(6.07,7.73)$ & $1.47(1.21,1.66)$ & 0.000 \\
\hline VAS score & $7.00(6.00,8.00)$ & $0.90(0.78,1.00)$ & 0.000 \\
\hline SAS score & $55.25(54.36,62.50)$ & $29.80(28.35,31.10)$ & 0.000 \\
\hline SDS score & $55.00(51.25,65.00)$ & $32.60(30.39,34.39)$ & 0.000 \\
\hline PF score & $50.00(33.75,55.00)$ & $62.82(59.75,66.68)$ & 0.000 \\
\hline RP score & $0.00(0.00,25.00)$ & $30.19(25.33,33.48)$ & 0.000 \\
\hline BP score & $50.00(50.00,62.00)$ & $74.98(71.47,79.48)$ & 0.000 \\
\hline GH score & $44.97 \pm 11.82$ & $58.73(54.68,62.03)$ & 0.000 \\
\hline VT score & $40.00(31.50,44.25)$ & $42.50(39.52,45.24)$ & 0.071 \\
\hline SF score & $55.00(37.50,62.50)$ & $59.87(57.58,62.49)$ & 0.439 \\
\hline RE score & $33.33(0.00,66.66)$ & $66.67(33.33,66.67)$ & 0.301 \\
\hline MH score & $45.00(42.00,61.00)$ & $66.38(63.98,69.06)$ & 0.000 \\
\hline ESR(mm/h) & $41.5(27.75,77.00)$ & $2.63(2.49,2.71)$ & 0.000 \\
\hline hs-CRP(mg/L) & $28.43(8.10,60.77)$ & $1.85 \pm 0.99$ & 0.000 \\
\hline RF(U/ml) & $91.85(23.45,255.15)$ & $4.56(4.45,4.64)$ & 0.000 \\
\hline CCP $(\mathrm{U} / \mathrm{ml})$ & $55(15.45,200.00)$ & $2.50 \pm 0.62$ & 0.000 \\
\hline
\end{tabular}




\section{Expression Of The Apoptosis-related Differential Proteins In Ra Patients}

To identify the apoptosis-related differential proteins in RA, we performed antibody microarrays analysis of the the apoptosis-related differential proteins in the PBMCs from four patients with RA and seven healthy people. After the raw data were normalized, the the apoptosis-related proteins were identified between the two groups. We identified 30 proteins (17 upregulated and 13 downregulated) that were differentially expressed between the two groups (Fig. 1A). Scatter plots showed 10 abundance of the apoptosis-related differential proteins by screening for log2-fold-changes greater than 1 and $\mathrm{P}<0.05$ (Fig. 1B). A heat map was constructed to group the the apoptosis-related differential proteins based on their expression levels among the samples (Fig. 1C). OPLS-DA clearly separated tested samples into two blocks according to their apoptosis-related differential proteins in both RA and healthy controls (Fig. 1D).

\section{Function of the apoptosis-related differential proteins in RA patients}

To explore the the apoptosis-related differential proteins, Gene Ontology (GO) terms, Kyoto Encyclopedia of Genes and Genomes (KEGG) and functional enrichment analyses were performed. We placed emphasis on $\mathrm{GO}$ analysis of proteins found that regulation of apoptotic process involved in morphogenesis, regulation of apoptotic process involved in deveiopment, positive regulation of proteolysis were in Biological Process (BP) (Fig. 2A), varicosity, receptor complex and neuronal cell body were in Cellular Component (CC) (Fig. 2B), TRAIL binding, death receptor binding, death receptor activity were in Molecular Function (MF) (Fig. 2C). Many pathways connected with functions of proteins in RA were defined by the KEGG analysis, TNF signaling pathway, MAPK signaling pathway, Apoptosis were in proteins (Fig. 2D).

\section{Protein-protein interaction network describing RA}

To better understand the cellular networks that are altered in the PBMCs of RA patients, the relationship between proteins was analyzed using different cutoff points with similar results (Fig. 3A). Additionally, we created protein-protein interaction network models of RA-specifc using the STRING database (Fig. 3B).

\section{Verification of the apoptosis-related differential proteins in RA patients}

A total of 10 the apoptosis-related differential proteins by screening for log2-fold-changes greater than 1 and $\mathrm{P}<0.05$ in RA (Table 2), including 7 upregulated and 3 downregulated proteins were selected.

Serum from 30 RA patients and 30 healthy controls were used for verification by ELISA. We selected 10 the apoptosis-related differential proteins from the most significant proteins for further verification. The expression profile of caspase3, CD40, SMAC $(p<0.01)$ in the RA group was apparently lower than in healthy control (Fig. 4A, B, G). The expression profile of HSP27, HTRA, IGFBP-1, IGFBP-6, sTNF-R1, sTNF$R 2$, TRAILR-3 $(P<0.01)$ in the RA group were apparently higher than healthy control (Fig. 4C, D, E, F, H, I, J). 
Table 2

Basic characteristics of the 10 the apoptosis-related differential proteins in RA patients.

\begin{tabular}{|lllll|}
\hline Protein name & Gene ID & Foldchange & $\mathbf{p}$ & Regulation \\
\hline HSP27 & 3315 & 4.656 & 0.000 & up \\
\hline IGFBP-1 & 3484 & 3.693 & 0.000 & up \\
\hline TRAILR-3 & 8794 & 0.326 & 0.000 & down \\
\hline STNF-R1 & 7132 & 0.390 & 0.000 & down \\
\hline CD40 & 958 & 1.664 & 0.003 & up \\
\hline STNF-R2 & 7133 & 0.531 & 0.003 & down \\
\hline HTRA & 27429 & 5.089 & 0.009 & up \\
\hline SMAC & 56616 & 3.033 & 0.011 & up \\
\hline IGFBP-6 & 3489 & 2.065 & 0.013 & up \\
\hline caspase3 & 836 & 2.667 & 0.017 & up \\
\hline
\end{tabular}

\section{ROC curve analysis of the apoptosis-related differential proteins in RA patients}

To further assess the biological functions, we performed ROC curve analysis to evaluate the diagnostic value of the 10 the apoptosis-related differential proteins. As showed in Fig. 5, ROC curves of confirmed proteins suggested that the levels of caspase3 (AUC $=0.9856), C D 40(A U C=1.0000), H S P 27(A U C=$ 0.8889), HTRA (AUC = 0.9988), IGFBP-1 (AUC = 1.0000), IGFBP-6 (AUC = 1.0000), SMAC $(A U C=1.0000)$, sTNF-R1 (AUC = 0.9744), sTNF-R2 (AUC = 0.9946), TRAILR-3 (AUC = 0.9917), which indicated that these proteins can be used as potential molecular markers for the diagnosis in RA.

Spearman Correlation analysis of the apoptosis-related differential proteins with SPP and clinical indexes in RA patients

To clarify the relationship between the the apoptosis-related differential proteins with SPP and clinical indexes, we performed Spearman Correlation analysis. The results of Spearman Correlation analysis suggested that caspase 3 was positively correlated with age, ESR, RF (Fig. 6A-C). HSP27 was negatively correlated with CCP (Fig. 6D). HTRA was positively correlated with VT (Fig. 6E). IGFBP-1 was positively correlated with SAS (Fig. 6F). IGFBP-6 was negatively correlated with VAS (Fig. 6G). SMAC was positively correlated with CRP, negatively correlated with RF (Fig. 6H-I). sTNF-R2 was positively correlated with ESR, negatively correlated with MH (Fig. 6J-K).

\section{Logistic-regression analysis of SPP, clinical indexes related the apoptosis-related differential proteins}

In order to identify risk factors of the the apoptosis-related differential proteins in RA patients, we conducted Logistic-regression analysis. Significant differences in caspase3 was found between RA 
patients with $\operatorname{ESR}(p=0.002), \operatorname{CCP}(p=0.027), \operatorname{SAS}(p=0.012)$, indicating that $\mathrm{ESR}, \mathrm{CCP}$, SAS were risk factors for caspase3. Significant differences in HPS27 was found between RA patients with VAS ( $p=$ $0.034), \mathrm{MH}(p=0.038)$, indicating that VAS and MH were risk factors for HPS27. Significant differences in SMAC was found between RA patients with $\operatorname{RF}(p=0.023)$, DAS28 $(p=0.050), \operatorname{VT}(p=0.001)$, indicating that RF, DAS28, VT were risk factors for SMAC.

\section{Discussion}

The early diagnosis of RA is very challenging because there are no specifc diagnostic indicator. Antibody microarray assay-based proteomics is a state-of-the-art analytical technique that enables the diagnosis and treatment of diseases. Proteomics has been a subject of interest in recent research, there has been a recent expansion in proteomics research on a number of diferent rheumatic diseases. Many factors contributed to decreased SPP for those living with $\mathrm{RA}^{25}$. With the change of medical model from a biomedical to a bio-psycho-social one and with the development of value-based medicine, SPP should be considered in making clinical decision ${ }^{26}$. Therefore, medical workers are increasingly concerned about SPP of RA. However, it is necessary to seek out new biomarkers and explore their functions for details of the mechanisms of decreased SPP in RA remain ambiguous.

Protein chips play an important role in scientific research due to its richer detect target factors and smaller sample size requirement. It is widely used in the research of disease mechanisms. Recently, Mun $S$ producted a analysis of changes in serum protein expression profiles of RA patients by SCIEX TripleTOF 560, which found that serum amyloid A4 and vitamin D binding protein could be potential biomarkers related to the inflammatory response and joint destruction that accompany RA. This finding provides an important reference for further research on RA ${ }^{27}$. To identify predictive biomarkers in patients with RA, 50 the apoptosis-related proteins profiling was conducted individually with 7 PBMCs from the normal group and 9 from the patient group with RA. Analysis of proteins profiling revealed the ten apoptosis-related proteins in the discovery set that could be considered as potential candidate biomarkers. The ten candidate biomarkers, namely cysteine aspastic acid-specific protease 3 (caspase3), heat shock protein 27 (HSP27), tumor necrosis factor receptor superfamily member 5 (TNFRSF5, CD 40), second mitochondria-derived activator of caspase, SMAC), HtrA serine peptidase 1 (HTRA), insulin-like growth factor binding protein - 1 (IGFBP-1), insulin-like growth factor binding protein - 6 (IGFBP-6), tumor necrosis factor receptor superfamily member $1 \mathrm{~A}$ (sTNF-R1), tumor necrosis factor receptor superfamily member 1B (sTNF-R2), tumor necrosis factor receptor superfamily member 10C (TRAILR-3) with > 1.5 of fold change in RA compared to healthy controls. Functional annotation of the the apoptosis-related proteins into GO terms and KEGG pathways was performed, which revealed that these the apoptosisrelated proteins involved in many pathophysiological processes, including regulation of apoptotic process, receptor complex, death receptor binding. A PPI network was constructed, which showed that these the apoptosis-related proteins are closely related in terms of their functions.

ELISA experiments for ten the apoptosis-related proteins were performed to further verify the diferential expression level. The expression profile of caspase3, CD 40, SMAC $(p<0.01)$ in the RA group was 
apparently lower than in healthy control. While the expression profile of HSP27, HTRA, IGFBP-1, IGFBP-6, sTNF-R1, sTNF-R2, TRAILR-3 $(P<0.01)$ in the RA group were apparently higher than healthy control. ROC curve analysis revealed the 10 the apoptosis-related differential proteins as diagnostic predictors of RA ( all AUC value is greater than 0.900). Caspase 3 is involved in signalling pathways leading to apoptosis, which plays significant roles in the pathogenesis of $\mathrm{RA}^{28}$. Hsps have been implicated in the RA ${ }^{29}$. Sedlackova L found that significantly increased Hsp27 and Hsp90a mRNA levels in RA synovial tissues, which indicated that Hsps could be new diagnostic approach to RA patients ${ }^{30}$. The anti-apoptotic activity of inhibitors of apoptosis proteins (IAP) proteins can be blocked by the SMAC, which is liberated into the cytoplasm in response to proapoptotic stimuli. Lattuada D found that SMAC was associated with caspase 8 and caspase 3 activities, and induced significant apoptosis in all RA-FLS samples. In addition, SMAC significantly upregulated IGFBP-5, a protein involved in differentiation, apoptosis, and osteoblastic activation, so they included that SMAC may represent a new therapeutic approach to RA treatment ${ }^{31}$.

Finally, we would like to discuss our findings in regard of their SPP and clinical indexes. We performed Spearman Correlation analysis suggested that caspase 3 was positively correlated with age, ESR, RF, HSP27 was negatively correlated with CCP, HTRA was positively correlated with VT, IGFBP-1 was positively correlated with SAS, IGFBP-6 was negatively correlated with VAS, SMAC was positively correlated with CRP, negatively correlated with RF, sTNF-R2 was positively correlated with ESR, negatively correlated with $\mathrm{MH}$. Additionally, results from Regression Analysis indicated that ESR, CCP, SAS were risk factors for caspase3, VAS and MH were risk factors for HPS27, RF, DAS28, VT were risk factors for SMAC. Liao $\mathrm{H}$ used 2-dimensional liquid chromatography-coupled tandem mass spectrometry to identify protein biomarkers of disease severity in the synovial fluid and serum of RA patients, they concluded that CRP, S100A8, S100A9 and S100A12 proteins could be identified for prognosis of the erosive form of RA ${ }^{32}$. However, there were no analysis between potential proteins and clinical indicators, nor did it predict the risk factors affectted potential proteins.

This study addresses important questions in understanding the important problem area in persons with decreased SPP in RA. The novelty in this study is that we have used microarray analysis combined with SPP to investigate their relationship. The main limitation of this study is a small number and inhomogeneity of respondents. Further in vitro and animal studies should be performed to evaluate comprehension of the detailed mechanism and specific functions of genes in decreased QOL in RA. To sum up, the ten apoptosis-related differential proteins are of potentially significant prediction value in decreased SPP in RA patients, but there remains a need to further study the mechanisms of these proteins.

\section{Conclusions}

In this work, we focus on the relationship between self-perception of patient (SPP) and the apoptosisrelated proteins in RA patients. We choosed four RA patients and seven healthy control for antibody array. Ten apoptosis-related proteins were verified by ELISA.We recruited 30 patients with RA and 30 healthy controls to fill in the five quentionnaires, including DAS28, VAS, SDS, SAS, SF-36. Finally, Spearman 
Correlation Analysis and Logistic-regression Analysis revealed that the ten apoptosis-related proteins were closely associated with SPP. They may serve as potential biomarkers for RA patient diagnosis.

\section{Abbreviations}

RA: rheumatoid arthritis; SPP:self-perception of patient; PBMCs:peripheral blood mononuclear cells; ELISA:Enzyme linked immunosorbent assay; ROC:Receiver operating characteristic; QOL:quality of life; DAS28:the Disease Activity Score in 28 joints; VAS:Visual Analogue Scale ; SDS:Self-rating Depression Scale; SAS:Self-rating Anxiety Scale; SF-36:MOS 36-Item Short Form Survey; CRP:high-sensitivity Creactive protein; ESR:erythrocyte sedimentation rate; RF:rheumatoid factor; CCP:anti-cyclic citrullinated peptide antibody; PF:physical functioning; RP:role-physical; BP:body pain; GH:general health; VT:vitality; SF:social functioning; RE:role-remotional; MH:mental health; caspase3:cysteine aspastic acid-specific protease 3; HSP27:heat shock protein 27; TNFRSF5:tumor necrosis factor receptor superfamily member 5; SMAC:second mitochondria-derived activator of caspase; HTRA:HtrA serine peptidase 1; IGFBP1:insulin-like growth factor binding protein - 1; IGFBP-6:insulin-like growth factor binding protein - 6; sTNF-R1:tumor necrosis factor receptor superfamily member 1A; sTNF-R2:tumor necrosis factor receptor superfamily member 1B; TRAILR-3:tumor necrosis factor receptor superfamily member 10C.

\section{Declarations}

\section{Authors'contributions}

WJT, LJ, JH, WL, SY, and XL contributed to the study design. WJT contributed to data analysis, wrote the first draft, and revised the manuscript. SYQ, ZY, DXL, WX, and WJ contributed to the specimen and date collection. LJ was the supervisors of the project and contributed to the manuscript revision. All authors reviewed and accepted the content of the final manuscript.

\section{Fundings}

This work was supported by grants from Ministry of Science and Technology National Key Research and Development Program Chinese Medicine Modernization Research Key Project (2018YFC1705204); National Nature Fund Program (81973655); The Key Research and Development Program Foreign Science and Technology Cooperation Project of Anhui (201904b11020011); Anhui Provincial quality Engineering Teaching and Research Project (2018jyxm1068); Anhui famous Traditional Chinese Medicine Liu Jian Studio Construction Project (Traditional Chinese Medicine Development Secret [2018] No. 11); National Key innovative talents training Project of Traditional Chinese Medicine (National Education letter of Traditional Chinese Medicine [2019] No.128); Key Research and Development Plan Project of Anhui Province (201904a07020004); Anhui Provincial Laboratory of Applied basis and Development of Internal Medicine of Modern Traditional Chinese Medicine (2016080503B041); 12th batch of "115" Innovation team of Anhui Province (Anhui Talent Office [2019] No. 1).

\section{Availability of data and materials}


The data used to support the findings of this study are available from the corresponding author upon request.

\section{Ethics approval and consent to participate}

The study was approved by the Ethics Committee of the First Affiliated Hospital of Anhui University of Traditional Chinese Medicine and carried out under the Helsinki Declaration. Before participating in the study, the patients filled in a written informed consent forms. A written informed consent was obtained from all the study participants.

\section{Consent for publication}

Not applicable.

\section{Conflicts of interest}

The authors have no competing interests to declare.

\section{References}

1. J W, S Y, J Y, et al. Non-coding RNAs in Rheumatoid Arthritis: From Bench to Bedside. 2019;10:3129. doi: 10.3389/fimmu.2019.03129.

2. WM G, evidence KJJBc. Rheumatoid arthritis: previously untreated early disease. 2016;2016.

3. VP N, KP M. G E, et al. Benefit of very early referral and very early therapy with disease-modifying anti-rheumatic drugs in patients with early rheumatoid arthritis. 2004;43(7):906-14. doi: 10.1093/rheumatology/keh199.

4. C SM. M, S B, et al. Rheumatoid arthritis treatment: the earlier the better to prevent joint damage. 2015;1:e000057. doi: 10.1136/rmdopen-2015-000057.

5. S N, Y T, M I, et al. Severe joint deformity and patient global assessment of disease are associated with discrepancies between sonographic and clinical remission: A cross-sectional study of rheumatoid arthritis patients. 2020:1-9. doi: 10.1080/14397595.2020.1751922.

6. $L Z, Y X, Q Z$, et al. The correlations of socioeconomic status, disease activity, quality of life, and depression/anxiety in Chinese patients with rheumatoid arthritis. 2017;22(1):28-36. doi: 10.1080/13548506.2016.1198817.

7. J J, L Z, Q Z, et al. Functional disability associated with disease and quality-of-life parameters in Chinese patients with rheumatoid arthritis. 2017;15(1):89. doi: 10.1186/s12955-017-0659-z.

8. L Z, J W, Q Z, et al. Factors associated with hand joint destruction in Chinese patients with rheumatoid arthritis. 2017;18(1):211. doi: 10.1186/s12891-017-1548-7.

9. Mcwilliams LA, Clara IP, Murphy PDJ, et al. Associations Between Arthritis and a Broad Range of Psychiatric Disorders: Findings From a Nationally. Representative Sample. 2008;9(1):37-44. 
10. Psychological affection in. rheumatoid arthritis patients in relation to disease activity: Erratum. \%J Medicine. 2019;98(28):e16515. doi:10.1097/md.0000000000016515.

11. MAH OV, Z DG. JWJ B, et al. International Consortium for Health Outcome Measurement Set of Outcomes That Matter to People Living With Inflammatory Arthritis: Consensus From an International Working Group. 2019;71(12):1556-65. doi: 10.1002/acr.23799.

12. M I GM. J C, et al. Quality of life in Ecuadorian patients with established rheumatoid arthritis. 2019;11:199-205. doi: 10.2147/oarrr.S216975.

13. MV N, DV Z, IV B, et al. Epigenetic Changes in the Pathogenesis of Rheumatoid Arthritis. 2019;10:570. doi: 10.3389/fgene.2019.00570.

14. C RM. Z, M A, et al. The emerging role of epigenetics in human autoimmune disorders. 2019;11(1):34. doi: 10.1186/s13148-019-0632-2.

15. R S, V R-E, chemistry HMJCtim. Rheumatoid arthritis: a clinical overview of new diagnostic and treatment approaches. 2013;13(6):698-704. doi: 10.2174/15680266113139990092.

16. O ES. S, G F, et al. Multiple biomarker approach for the diagnosis and therapy of rheumatoid arthritis. 2020:1-12. doi: 10.1080/10408363.2020.1775545.

17. L M-P MM. JA S, et al. Precision medicine in the care of rheumatoid arthritis: Focus on prediction and prevention of future clinically-apparent disease. 2020;19(5):102506. doi:

10.1016/j.autrev.2020.102506.

18. EJ K, BA dJ, MA vL, et al. The prognostic value of anti-cyclic citrullinated peptide antibody in patients with recent-onset rheumatoid arthritis. 2000;43(8):1831-5. doi: 10.1002/1529-

0131(200008)43:8<1831::Aid-anr19>3.0.Co;2-6

19. A PZ. R, SA E, et al. Survivin a pivotal antiapoptotic protein in rheumatoid arthritis. 2019;234(12):21575-87. doi: 10.1002/jcp.28784.

20. Q Z. J L, M Z, et al. Apoptosis Induction of Fibroblast-Like Synoviocytes Is an Important MolecularMechanism for Herbal Medicine along with its Active Components in Treating Rheumatoid Arthritis. 2019;9(12) doi: 10.3390/biom9120795.

21. J Y, E W, cancer FBJJfio. Immune Monitoring Technology Primer: protein microarray ('seromics'). 2016;4:2. doi: 10.1186/s40425-016-0106-4.

22. Ng JH, Ilag LLJJoC, Medicine M. Biomedical applications of protein chips. 2007;6(3):329-40.

23. BH WH. T, X Z, et al. Proteomic analysis of secreted proteins in early rheumatoid arthritis: anticitrulline autoreactivity is associated with up regulation of proinflammatory cytokines. 2007;66(6):712-9. doi: 10.1136/ard.2006.054924.

24. T DA. N, AJ S, et al. 2010 Rheumatoid arthritis classification criteria: an American College of Rheumatology/European League Against Rheumatism collaborative initiative. 2010;62(9):2569-81. doi: 10.1002/art.27584.

25. Englbrecht M, Kruckow M, Araujo E, et al. The Interaction of Physical Function and Emotional Wellbeing in Rheumatoid Arthritis-What is the Impact on Disease Activity and Coping? 2013;42(5):482 - 
91.

26. Dr, Anthony S, et al. Quality-of-Life Assessment in Rheumatoid Arthritis. 2008.

27. S M, J L, A P, et al. Proteomics Approach for the Discovery of Rheumatoid Arthritis Biomarkers Using Mass Spectrometry. 2019;20(18) doi: 10.3390/ijms20184368.

28. S L, S W, Z Z, et al. MiRNA-6089 inhibits rheumatoid arthritis fibroblast-like synoviocytes proliferation and induces apoptosis by targeting CCR4. 2020:1-8. doi: 10.1080/13813455.2020.1773862.

29. AK RK, HMN M. I, et al. Heat Shock Proteins: Therapeutic Perspectives in Inflammatory Disorders. 2017;10(2):94-104. doi:10.2174/1872213x10666161213163301.

30. L S, A S, P V, et al. Heat shock protein gene expression profile may differentiate between rheumatoid arthritis, osteoarthritis, and healthy controls. 2011;40(5):354-7. doi:

10.3109/03009742.2011.552522.

31. C DL. C, K C, et al. Proapoptotic activity of a monomeric smac mimetic on human fibroblast-like synoviocytes from patients with rheumatoid arthritis. 2015;38(1):102-9. doi: 10.1007/s10753-0140012-1.

32. H L, J W, E K, et al. Use of mass spectrometry to identify protein biomarkers of disease severity in the synovial fluid and serum of patients with rheumatoid arthritis. 2004;50(12):3792-803. doi: 10.1002/art.20720.

\section{Figures}

A

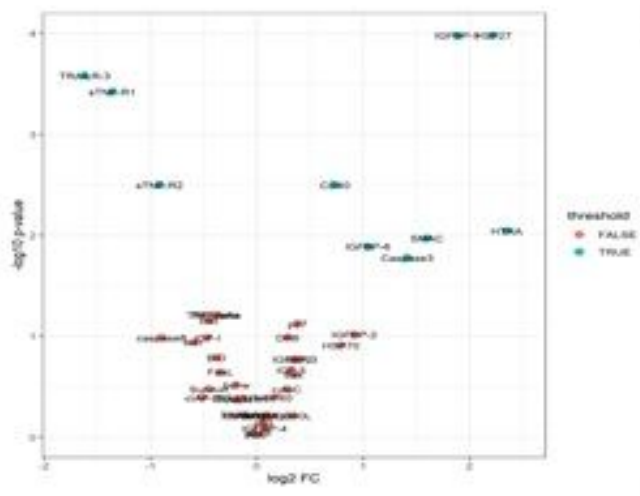

$\mathrm{C}$

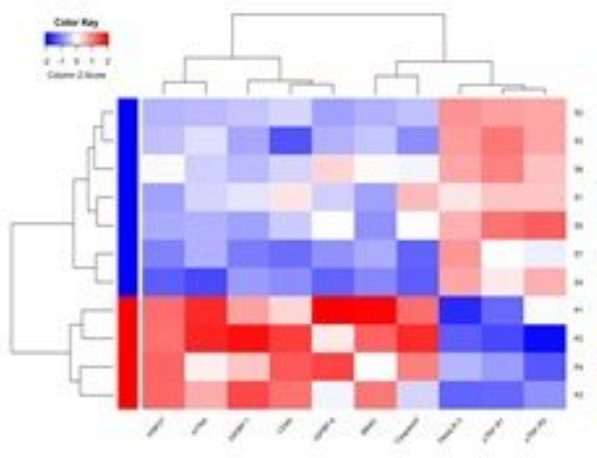

B

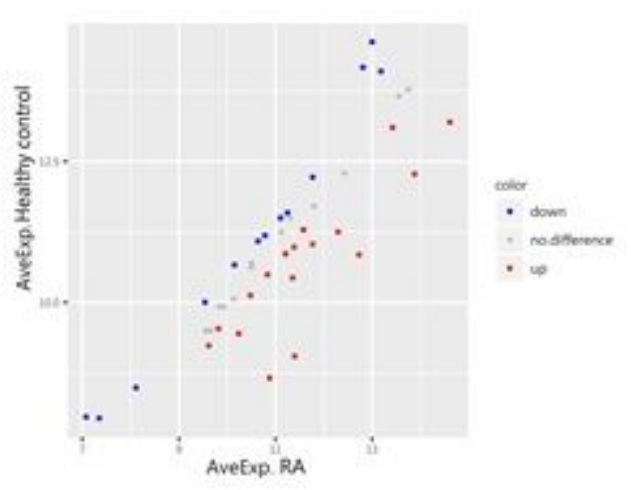

D

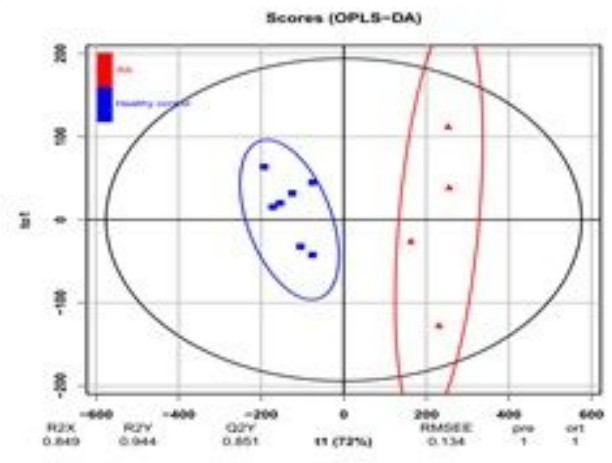

Figure 1 
Antibody microarrays determining the apoptosis-related differential proteins expression profiles in RA patients. (A) Volcano plot of the apoptosis-related differential proteins: The vertical lines correspond to 1.5-fold up and down and the horizontal line represents a P-value of 0.05 , blue: true; red: false. (B) Scatter plot of the apoptosis-related differential proteins: Red: up expression; blue: down expression; grey: no difference. (C) Heatmap of the apoptosis-related differential proteins: Red: high relative expression; blue: low relative expression. (D) Principal component analysis of the apoptosis-related differential proteins: Red: RA, blue: healthy controls.

A

$\mathrm{C}$
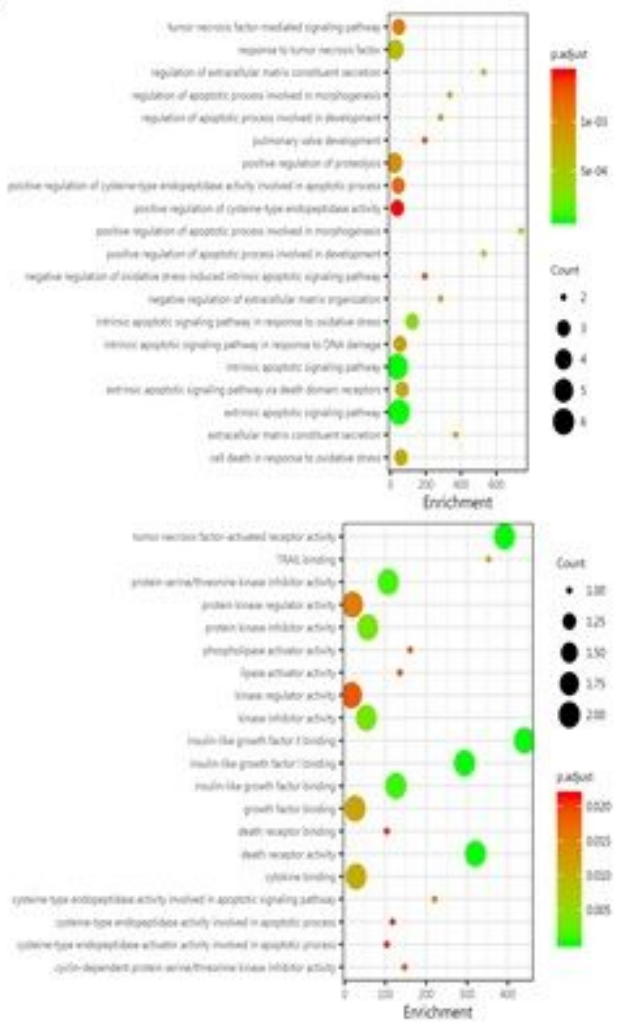

B

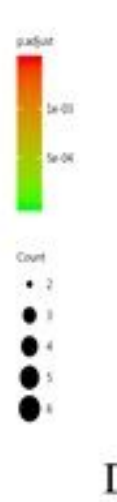

$\mathrm{D}$
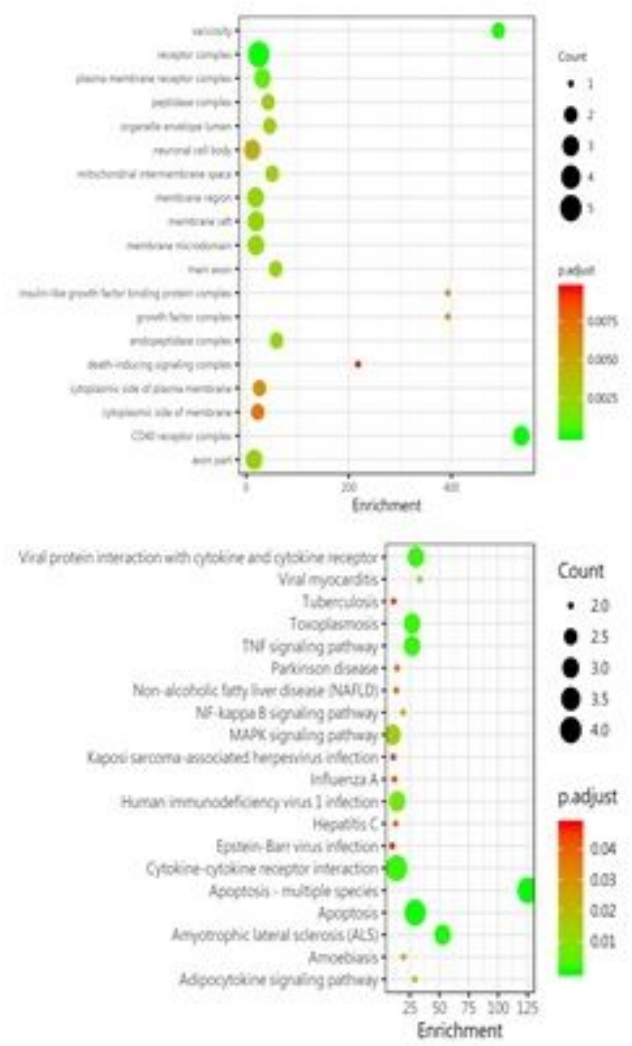

Figure 2

GO analysis and KEGG pathway annotation of the apoptosis-related differential proteins. (A) The top 20 significantly obvious changes of BP in GO biological process classification of proteins. (B) The top 19 significantly obvious change of $\mathrm{CC}$ in $\mathrm{GO}$ biological process classification of proteins. (C) The top 20 significantly obvious changes of MF in GO biological process classification of proteins. (D) The top 20 significant obvious changes in KEGG pathway classification of proteins. The bar plot shows the top Enrichment Score (-log10 (Pvalue)) value of the significant pathway. The color represents the difference, darker indicating a greater difference. The circle represents the relationship. 
A

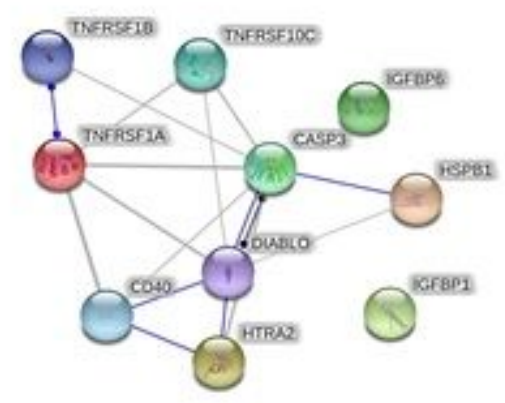

B

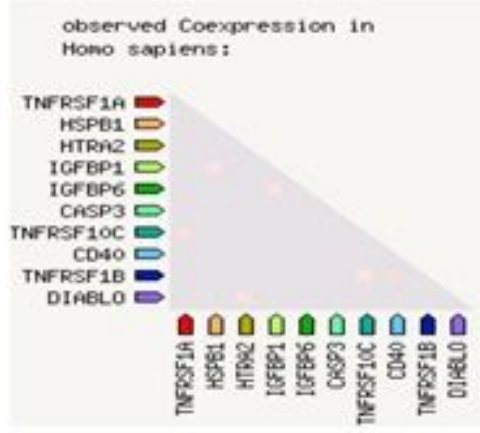

observed Coexpression in other organishs (transferred):

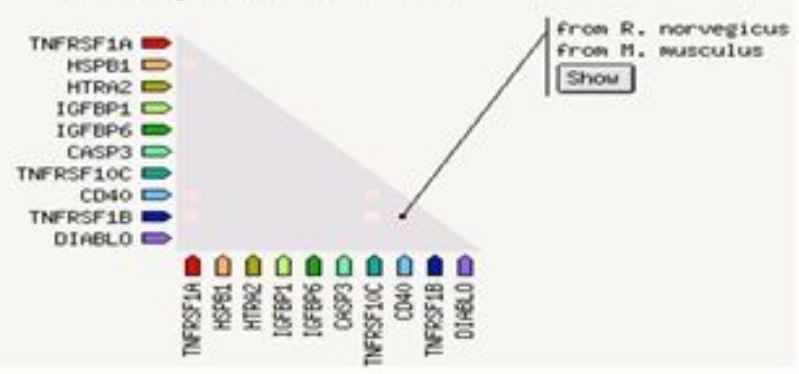

\section{Figure 3}

Interaction network of the the apoptosis-related differential proteins. (A) The relationship between differential proteins. (B) The PPI network of the the apoptosis-related differential proteins was constructed. 
A

B

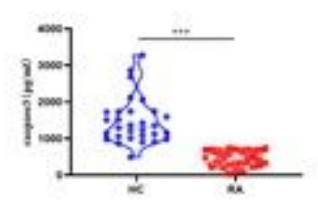

E

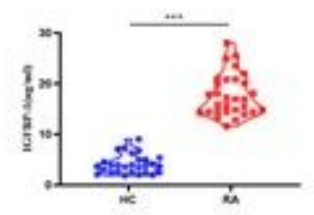

F

I

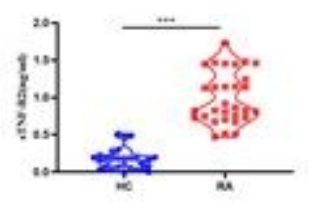

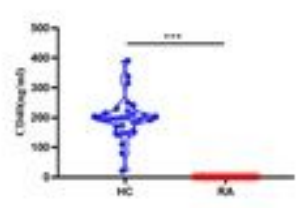

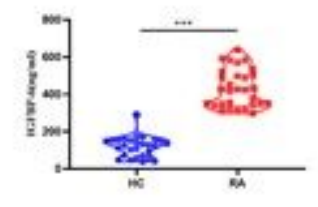

C

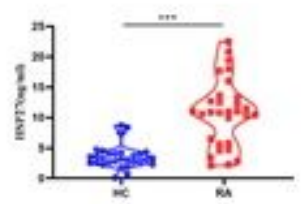

G

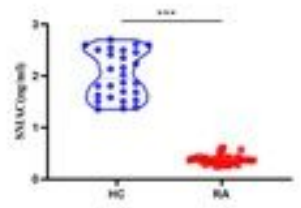

J

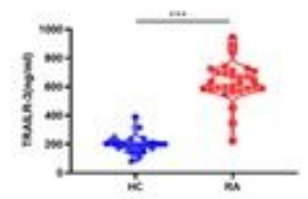

D

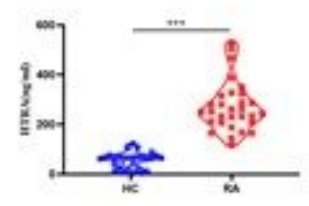

H

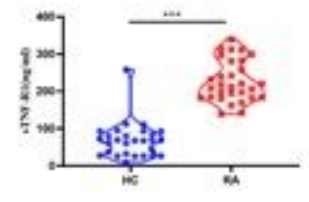

Figure 4

Serum levels of the the apoptosis-related differential proteins were measured by ELISA. (A, B, G) Caspase3, CD 40, SMAC expression were significantly decreased ( $P<0$.01). (C, D, E, F, H, I, J) HSP27, HTRA, IGFBP-1, IGFBP-6,sTNF-R1, sTNF-R2, TRAILR-3 expression were significantly increased $(P<0.01)$.

Page $18 / 20$ 
A

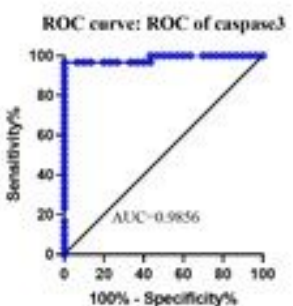

E

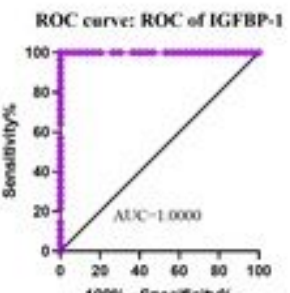

I

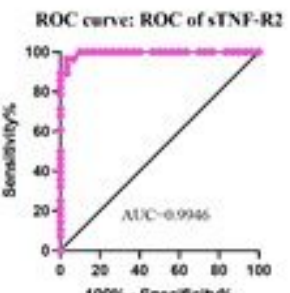

B

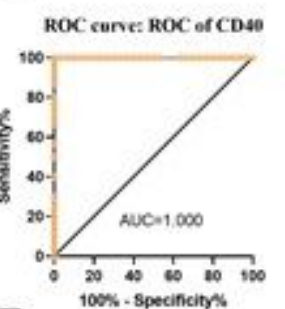

F

ROC curve: ROC of IGFBP.6

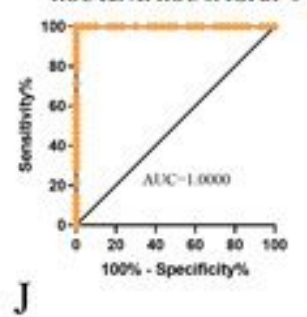

ROC cune: ROC of TRAILR-3

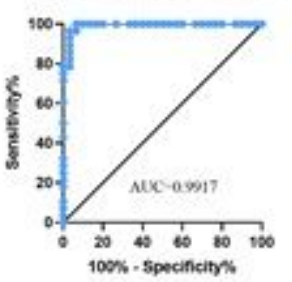

C

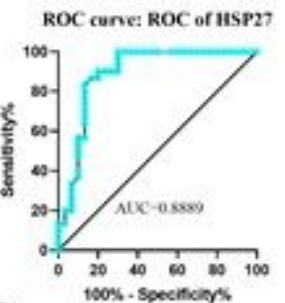

G

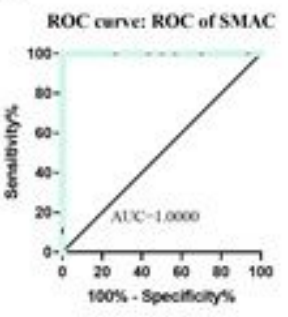

D

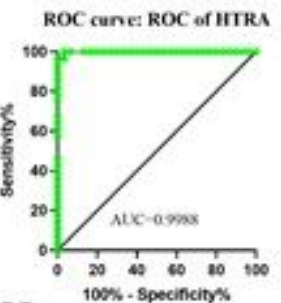

$\mathrm{H}$

ROC cune: ROC of STNF-RI

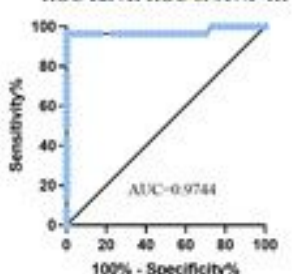

Figure 5

ROC curve analysis of the apoptosis-related differential proteins.

A

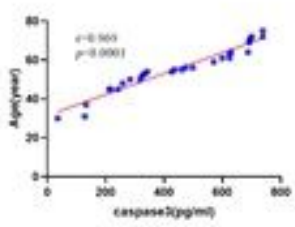

$\mathrm{E}$

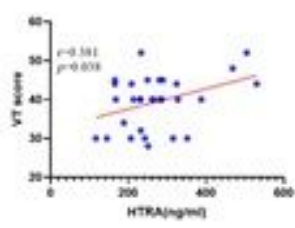

I

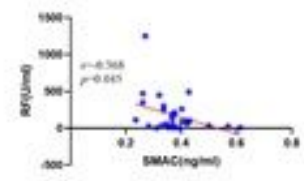

B

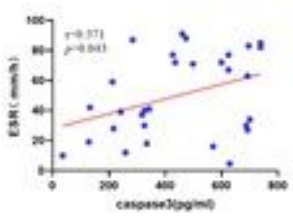

$\mathrm{F}$

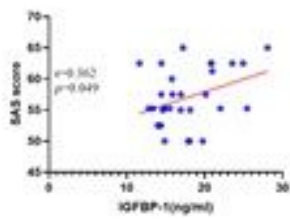

J

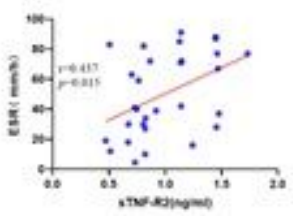

C

D

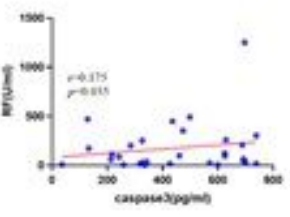

G

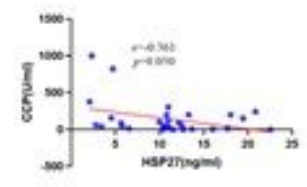

$\mathrm{H}$
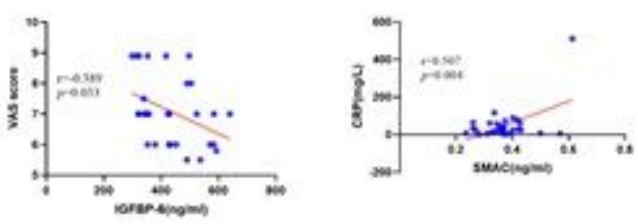

K

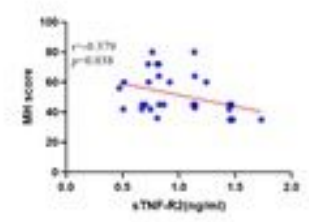




\section{Figure 6}

Correlation between the apoptosis-related differential proteins with SPP and clincial indexes. (A - C) There was a close positive correlation of the caspase3 with age, ESR, RF. (D) There was a close negative correlation of the HSP27 with CCP. (E) There was a close positive correlation of the HTRA with VT. (F) There was a close positive correlation of the IGFBP-1 with SAS. (G) There was a close negative correlation of the IGFBP-6 with VAS. (H-I) There was a close positive correlation of the SMAC with CRP, a close negative correlation of the SMAC with RF. (K) There was a close positive correlation of the sTNF-R2 with ESR, a close negative correlation of the sTNF-R2 with MH.

A

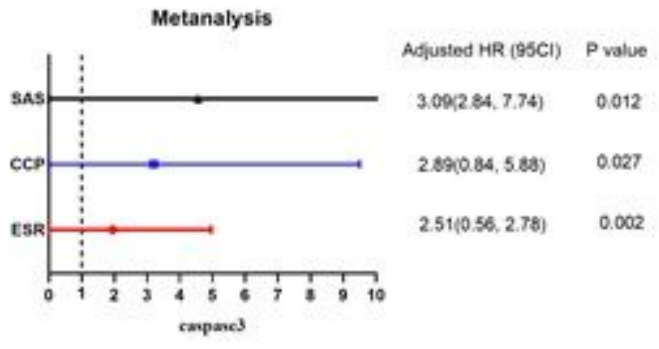

$\mathrm{C}$

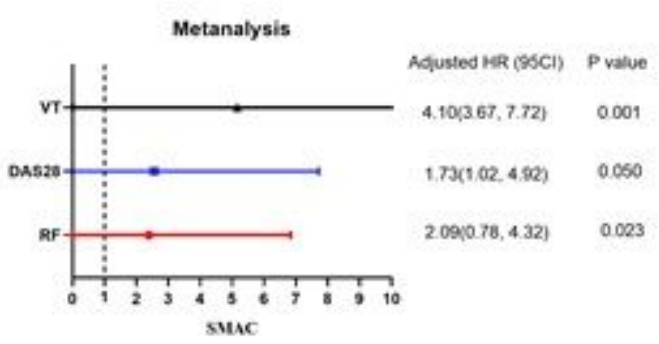

B

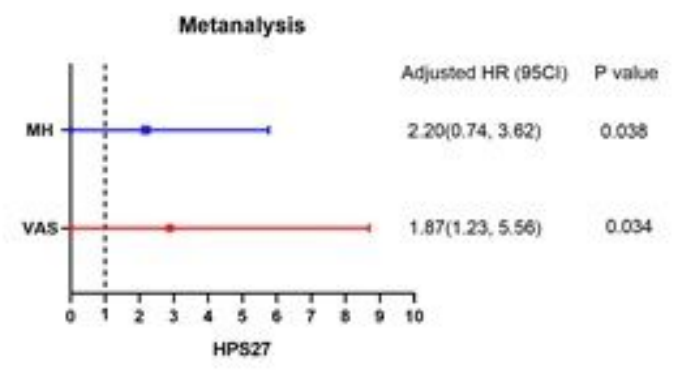

\section{Figure 7}

Logistic Regression analysis of the apoptosis-related differential proteins related factors. (A) ESR, CCP, SAS were risk factors for caspase3. (B) VAS and MH were risk factors for HPS27. (C) RF, DAS28, VT were risk factors for SMAC. 